\title{
CORRELATION BETWEEN MUSCLE STRENGTH AND ENDURANCE WHEN USING SPECIALIZED FITNESS TRAINING FOR STUDENTS
}

\author{
P. Petkov* \\ Trakia University, Stara Zagora, Bulgaria
}

\begin{abstract}
Testing was conducted during the winter semester of the academic 2016/2017 year. A total of 67 students from the Faculties of Agriculture, Veterinary Medicine and Economics at Trakia University , Stara Zagora, are the contingent of the survey. The average age of the surveyed students was 20.04 years.

The aim of the study was to establish a correlation between muscle strength and endurance as a result of applying a specialized fitness model. To achieve this goal, the following tasks were assigned: review of scientific literature, development and approbation of a specialized fitness model, development of a test battery, processing and analysis of the data from the conducted testing. The following methods were applied: pedagogical experiment, testing and correlation analysis. Based on the conducted detailed correlation analysis, we believe that following the experimental fitness program, the relationships between muscle strength and stamina retain their essential characteristic - with a significant number of reliable statistical links; after the completion of the experiment, the statistically significant correlations of the EG increased. Significant increase with $99 \%$ statistical significance between motor skills after the application of the author's fitness model was observed; the conducted study showed different degrees of correlation dependencies between the motor skills muscle strength and stamina of the students.
\end{abstract}

Key words: University, experiment

\section{INTRODUCTION}

Modern lifestyles, coupled with scientific advances of technology, enter into a paradoxical contradiction with the person's functional capabilities. The mobilization of man enters more and more in his everyday life. This in turn leads to health problems - weight gain, increased cardiac arrest, decreased physical fitness and a number of other negative effects affecting the body.

The issue of physical development and functional abilities of students is becoming more and more relevant. Universities with their organized forms of physical education are the main factor in the development of students' physical capacity. The issue of improving and updating curricula in physical education and

*Correspondence to: Plamen Petkov, Trakia University, Stara Zagora, Bulgaria,

e-mail: jo_team@abv.bg, GSM 0887881260 sports classes is at the forefront. The main methodical problem is the application of innovative methods to optimize the training impacts in the training process, in accordance with the regularities of the adaptation process (1-3).

Many authors consider that basically the performance of motor tasks depends on the functional state of man. Muscle strength and endurance are an essential part of man's physical capacity. Silence as a physical quantity of a person is at the moment of motion. Promoting the power of its defenses, we are committed to doing everyday business, as well as re-establishing ourselves in the extreme of our business. It is the basis on which the partner technician and the sponsor are involved in the development of the spontaneous achievements (4-8). Many specialists have a special focus on the durability of physical insight in all age groups. This is associated with the fact that durability 
provides effective solutions to life-threatening systems in the human body: cardiovascular, respiratory, blood and osteoarthritis (9-12).

The aim of the study is to establish the correlation between muscle strength and endurance as a result of applying a specialized fitness model.

To achieve our goal, we have set the following tasks:

1. Study of literary sources

2. Development and approbation of a specialized fitness model.

3. Develop a test battery.

4. Process and analyze the test data.

Methods of study:

1. Pedagogical experiment

2. Testing

3. Correlation analysis

Testing was conducted during the winter semester of the school year 2016/2017. A total of 67 students from the Faculty of Agriculture, Faculty of Veterinary Medicine and Faculty of Economics at the Thracian University in Stara Zagora are part of the survey. The researchers surveyed had an average age of 20.04 years.

The test battery includes the following tests:

1. Jump length of place

2. Vertical jump

3. Deadlift

4. Dynamometry

5. Hanging to a denial

6. Dumbbell hold $2 \mathrm{~kg}$

7. Plank

8. Throwing the medical unit $(3 \mathrm{~kg}$ ) forward

9. Tossing of the medical unit $(3 \mathrm{~kg})$ back

10. Shuttle $10 \times 10 \mathrm{~m}$
11. Pushups to denial

12. Abdominal presses to denial

13. Harvard Stap Test - pulse increase during the step test

14. Harvard Steppe Test - Pulse after 1 minute

15. Harvard Steppe Test - Pulse after 3 minutes

16. Harvard Step Test - Pulse Time - 3 Minutes of Restoration

17. Cooper Test

18. Beep test

Results analysis:

The developed model includes tasks divided into muscular groups (for upper limbs, lower limbs, back, chest, abdominal muscles) with a certain degree of success and prestige. The trainee gymnast was offered for 24 weeks (twice a week) during the winter and summer semester of the 2016/2017 school year.

At the time of the experiment, the students carried out the assignments, assigned in three different circles. Each round runs for 8 weeks with a gap between two minutes in the first four weeks and one minute during the four weeks. Each of the circles contained 15 exercises. The duration of one exercise is 15 seconds. The fifteen performances in the circle are carried out without a prank between them. Every round of the round is completed five times.

The absolute value of the correlations between observed mobility attributes and extent of their significance at the beginning and end of the experimental period of experimentation and organizational group is presented in the context of the corridor (Tables 1, 2, 3, 4).

Table 1._Correlation analysis KG first statement

\begin{tabular}{|c|c|c|c|c|c|c|c|c|c|c|c|c|c|c|c|c|c|c|}
\hline \multicolumn{7}{|c|}{ Jump length of ertical jum Deadlift mnamometsing to a dabbell hold } & \multirow{2}{*}{$\begin{array}{l}\text { Plank } \\
0,026\end{array}$} & \multirow{2}{*}{\begin{tabular}{|c|} 
redical un \\
0,135
\end{tabular}} & \multicolumn{10}{|c|}{ 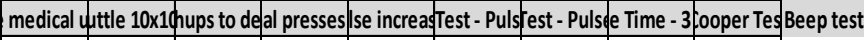 } \\
\hline Jump length of place & 1 &, $656 * *$ & 0,341 &, $379 *$ & ,413* & 0,023 & & & 0,188 & $-0,018$ & 0,066 & 0,238 & $-0,001$ & 0,046 & 0,28 & 0,282 & $-0,058$ & 0,13 \\
\hline Vertical jump & & 1 & 0,276 &, $405^{*}$ & 0,282 & $-0,036$ & 0,192 & 0,185 & 0,109 & $-0,283$ & 0,186 & 0,247 & $-0,049$ & 0,094 & 0,291 & 0,243 & $-0,039$ & 0,072 \\
\hline Deadlift & & & 1 & 0,306 & 0,169 & 0,005 &, $438^{*}$ & 0,096 & 0,046 & $-0,232$ & $-0,11$ & 0,151 & 0,226 & $-0,154$ & $-0,054$ & 0,185 & 0,021 & 0,017 \\
\hline Dynamometry & & & & 1 & 0,094 & 0,045 & 0,11 & 0,09 & 0,131 & 0,029 & 0,1 & 0,129 & 0,127 & $-0,162$ & 0,176 & 0,314 & 0,155 & $-0,1$ \\
\hline Hanging to a denial & & & & & 1 & 0,197 & 0,291 &, $364^{*}$ &, $418^{*}$ & $-0,095$ & $-0,056$ & $-0,075$ & 0,259 & 0,026 & 0,026 & 0,302 & $-0,27$ & 0,086 \\
\hline Dumbbell hold 2kg & & & & & & 1 & 0,146 &, $434^{*}$ & 0,326 & 0,125 & $-0,022$ & $\begin{array}{ll}-0,241 \\
\end{array}$ & 0,253 & 0,057 & 0,054 & 0,324 & 0,039 & 0,011 \\
\hline Plank & & & & & & & 1 & 0,219 & , $388^{*}$ & $-0,291$ &,$- 393 *$ & $-0,149$ & 0,128 & $-0,188$ & $-0,152$ & $-0,018$ & $-0,311$ & $-0,131$ \\
\hline \multicolumn{3}{|c|}{ Throwing the medical unit $(3 \mathrm{~kg})$ forward } & & & & & & 1 & , $534 * *$ & $-0,334$ & $-0,093$ & $-0,334$ & 0,252 & 0,104 & $-0,246$ & 0,019 & 0,106 & 0,169 \\
\hline \multicolumn{3}{|c|}{ Tossing of the medical unit (3kg) back } & & & & & & & 1 & 0,122 & $-0,343$ & $-0,086$ & 0,18 & $-0,112$ & $-0,145$ & 0,044 & $-0,063$ & 0,084 \\
\hline Shuttle $10 \times 10 \mathrm{~m}$ & & & & & & & & & & 1 & 0,189 & 0,044 & $-0,027$ & $-0,031$ & 0,208 & 0,182 & 0,066 & $-0,074$ \\
\hline Pushups to denial & & & & & & & & & & & 1 & 0,102 & $-0,045$ &, $386^{*}$ & 0,154 & 0,107 & 0,128 & 0,046 \\
\hline \multicolumn{2}{|c|}{ Abdominal presses to denial } & & & & & & & & & & & 1 & 0,093 & $-0,198$ & 0,248 &, $351^{*}$ & 0,302 & 0,172 \\
\hline \multicolumn{5}{|c|}{ Harvard Stap Test - pulse increase during the step test } & & & & & & & & & 1 & $-0,263$ &,$- 537^{* *}$ & ,519** & 0,066 & 0,155 \\
\hline \multicolumn{4}{|c|}{ Harvard Steppe Test - Pulse after 1 minute } & & & & & & & & & & & 1 & 0,097 & $-0,181$ & $-0,13$ & $-0,049$ \\
\hline \multicolumn{4}{|c|}{ Harvard Steppe Test - Pulse after 3 minutes } & & & & & & & & & & & & 1 &, $442^{*}$ & $-0,171$ & $-0,204$ \\
\hline \multicolumn{5}{|c|}{ Harvard Step Test - Pulse Time - 3 Minutes of Restoration } & & & & & & & & & & & & 1 & $-0,103$ & $-0,041$ \\
\hline Cooper Test & & & & & & & & & & & & & & & & & 1 &, $569 * *$ \\
\hline Beep test & & & & & & & & & & & & & & & & & & 1 \\
\hline
\end{tabular}

**. Correlation is significant at the 0.01 level (2-tailed).

*. Correlation is significant at the 0.05 level (2-tailed). 
Table 2._Correlation analysis $K G$ second statement

\begin{tabular}{|c|c|c|c|c|c|c|c|c|c|c|c|c|c|c|c|c|c|c|}
\hline \multicolumn{3}{|c|}{ Jump length of Ertical jur } & \multicolumn{4}{|c|}{ Deadlift mamomefing to ad bbell hold } & \multirow{2}{*}{$\begin{array}{l}\text { Plank } \\
-0,003 \\
\end{array}$} & \multirow{2}{*}{\begin{tabular}{|c} 
redica uni \\
0,062 \\
\end{tabular}} & \multicolumn{2}{|c|}{ medical uttle 10xu } & \multicolumn{2}{|c|}{ hups to deal presses } & \multirow{2}{*}{\begin{tabular}{|c|} 
sse increa \\
$-0,167$ \\
\end{tabular}} & \multicolumn{2}{|c|}{ Test - Pulstest - Pus s } & \multicolumn{2}{|c|}{ eTime-3 ooper Tes } & Beep tes \\
\hline Jump length of placi & 1 & $424^{*}$ & 0,299 & 0,272 &, $371^{*}$ & 0,077 & & & 0,253 & $-0,091$ & 0,02 & 0,098 & & 0,003 & $-0,239$ &,$- 378^{*}$ & $-0,002$ & 0,002 \\
\hline Vertica jump & & 1 & 0,043 & 0,054 &, $502 *$ & $-0,023$ & ,349* & 0,06 & 0,185 &,$- 373^{*}$ & $-0,046$ & 0,051 & 0,182 & $-0,137$ & $-0,175$ & $-0,015$ & 0,137 & 0,241 \\
\hline Deadlift & & & 1 & 0,305 & 0,135 & 0,16 &, $364^{*}$ & $-0,095$ & $-0,076$ & $-0,296$ & $-0,104$ & $-0,078$ & 0,158 & $-0,142$ & $-0,301$ & $-0,159$ & 0,229 & $-0,156$ \\
\hline Dynamometry & & & & 1 & $-0,05$ & 0,28 & 0,093 & 0,014 & 0,134 & 0,085 & 0,106 & 0,158 & $-0,178$ & $-0,154$ & $-0,061$ & $-0,214$ & 0,247 & 0,057 \\
\hline Hanging to adenia & & & & & 1 & 0,211 & 0,329 & 0,025 & , 350* & $-0,216$ & $-0,065$ & $-0,048$ & 0,164 & 0,084 & $-0,043$ & 0,099 & $-0,04$ & $-0,174$ \\
\hline Dumbbell hold $2 \mathrm{~kg}$ & & & & & & 1 & 0,339 & 0,274 & 0,261 & $-0,175$ & $-0,009$ & $-0,015$ & 0,32 & $-0,028$ & $-0,221$ & 0,06 & 0,206 & $-0,167$ \\
\hline Plank & & & & & & & 1 & 0,145 & 0,085 & $-459^{* *}$ & $-440^{*}$ & $-0,193$ & ,523** & $-0,299$ & $-447^{* *}$ & 0,013 & $-0,028$ & $-0,091$ \\
\hline \multicolumn{3}{|c|}{ Throwing the medical unit ( $3 \mathrm{~kg}$ ) forward } & & & & & & 1 &, $433^{*}$ & $-3,345^{*}$ & $-0,195$ & $-0,124$ & 0,149 & 0,137 & $-0,145$ & $-0,013$ & 0,17 & 0,106 \\
\hline \multicolumn{3}{|c|}{ Tossing of the medical unit (3kg) back } & & & & & & & 1 & $-0,142$ & $-0,202$ & $-0,074$ & 0,118 & 0,012 & $-0,3$ & $-0,193$ & 0,066 & 0,109 \\
\hline Shuttle $10 \times 10 \mathrm{~m}$ & & & & & & & & & & 1 & 0,239 & 0,034 &,$- 413^{*}$ & 0,005 &, $394^{*}$ & 0,03 & $-0,262$ & $-0,133$ \\
\hline Pushups to denia & & & & & & & & & & & 1 & 0,219 & $-403^{*}$ & $432^{*}$ & 0,244 & $-0,108$ & 0,037 & $-0,077$ \\
\hline \multicolumn{2}{|c|}{ Abdomina presses to denial } & & & & & & & & & & & 1 & $-0,25$ & $-0,131$ & 0,19 & $-0,03$ & $-0,064$ & 0,091 \\
\hline \multicolumn{5}{|c|}{ Hanard Stap Test - pullse increase during the step test } & & & & & & & & & 1 & $-0,305$ & $-417^{*}$ & ,453** & $-0,021$ & 0,087 \\
\hline \multicolumn{4}{|c|}{ Hanard Steppe Test - Pulse after 1 minute } & & & & & & & & & & & 1 & $434^{*}$ & 0,163 & $-0,06$ & $-0,336$ \\
\hline \multicolumn{4}{|c|}{ Hanvard Steppe Test - Pulse after 3 minutes } & & & & & & & & & & & & 1 &, $621^{* *}$ & $-0,192$ & $-414^{*}$ \\
\hline \multicolumn{5}{|c|}{ Hanard Step Test - Pulse Time - 3 Minutes of Restoration } & & & & & & & & & & & $621 * *$ & 1 & $-0,207$ & $-0,332$ \\
\hline CooperTest & & & & & & & & & & & & & & & & & 1 & ,452** \\
\hline Beeptest & & & & & & & & & & & & & & & & & & 1 \\
\hline
\end{tabular}

**. Correlation is significant at the 0.01 level (2-tailed).

* Correlation is significant at the 0.05 level (2-tailed).

Table 3._Correlation analysis EG first statement

\begin{tabular}{|c|c|c|c|c|c|c|c|c|c|c|c|c|c|c|c|c|c|c|}
\hline Jump l & nggth & 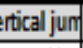 & Deadlift & namome & toat & bbell holg & Plank & 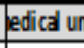 & - & $1010 x$ & pstod & presse. & se increa & est - Pul & Test - Puls & Time- & ooper Te & Beep test \\
\hline Jump length of plact & 1 & $645^{* *}$ & 0,265 &, $376^{*}$ & $496^{* *}$ & $-0,152$ & 0,115 & 0,066 & 0,16 & $-0,182$ & $-0,033$ & 0,036 & $-0,096$ & $-0,151$ & $-0,112$ & $-0,252$ & 0,198 & 0,18 \\
\hline Vertical jump & & 1 & , 336" & 0,299 &, $356^{*}$ & 0,171 & , $473^{* *}$ & 0,129 & 0,144 &,$- 362^{*}$ & 0,144 & 0,25 & 0,092 & $-0,197$ & $-0,159$ & $-0,031$ & 0,147 & 0,306 \\
\hline Deadlift & & & 1 & $526^{4 *}$ & $-0,097$ & 0,198 & $465^{* *}$ & 0,118 & 0,157 & $-3,368^{*}$ & 0,307 & 0,26 & $-0,077$ & $-0,123$ & 0,108 & 0,001 & $420^{*}$ & 0,199 \\
\hline Dynamometry & & & & 1 & 0,03 & 0,001 & 0,287 & 0,11 & 0,24 & $-0,202$ & 0,212 & 0,129 & $-0,208$ & 0,107 & 0,115 & $-0,179$ & 0,215 & 0,065 \\
\hline Hanging to adenia & & & & & 1 & $-0,098$ & 0,226 & 0,227 & 0,005 & $-0,007$ & $-0,065$ & $-0,003$ & $-0,13$ & 0,23 & $-0,062$ & $-0,25$ & $-0,112$ & 0,072 \\
\hline Dumbbell hold $2 \mathrm{~kg}$ & & & & & & 1 & 0,281 & 0,307 & 0,122 & $-0,004$ & $333^{*}$ & 0,178 & 0,265 & 0,083 & $-0,109$ & 0,268 & 0,326 & 0,203 \\
\hline Plank & & & & & & & 1 & 0,154 & 0,096 & $-4399^{* *}$ & 0,243 &, $349^{\circ}$ & $-0,245$ & 0,004 & 0,272 & $-0,073$ & 0,209 & 0,284 \\
\hline \multicolumn{3}{|c|}{ Throwing the medica unit ( $3 \mathrm{~kg}$ ) forward } & & & & & & 1 &, $623^{* 4}$ & $-0,326$ & $-0,078$ & $-0,165$ & $-0,186$ & $-0,113$ & 0,021 & $-0,246$ &, $573^{* *}$ &, $336^{*}$ \\
\hline \multicolumn{3}{|c|}{ Tossing of the medical unit (3kg) back } & & & & & & & 1 & $-0,042$ & $-0,188$ & $-0,198$ & $-0,068$ & $-0,131$ & $-0,068$ & $-0,196$ &, $344^{4}$ & $332^{*}$ \\
\hline Shuttle $10 \times 10 \mathrm{~m}$ & & & & & & & & & & 1 & $-0,178$ & $-0,157$ & 0,166 & 0,256 & $-0,097$ & 0,138 &,$- 394^{4}$ & $-0,193$ \\
\hline Pushups to denia & & & & & & & & & & & 1 & 0,09 & $-0,051$ & 0,081 & 0,187 & 0,118 & 0,155 & $-0,01$ \\
\hline \multicolumn{2}{|c|}{ Abdominal presses to denial } & & & & & & & & & & & 1 &, $378^{*}$ & $-0,08$ & $-0,011$ &, $530^{* *}$ & $-0,072$ & 0,073 \\
\hline \multicolumn{5}{|c|}{ Harvard Stap Test - pulse increase duning the step test } & & & & & & & & & 1 & 0,005 & $-7,716^{* *}$ & $6996^{* *}$ & $-0,3$ & $-0,046$ \\
\hline \multicolumn{4}{|c|}{ Harvard Steppe Test - Pulse d ter 1 minute } & & & & & & & & & & & 1 & 0,118 & 0,13 & $-0,267$ & $-0,137$ \\
\hline \multicolumn{4}{|c|}{ Harvard Steppe Test - Pulse after 3 minutes } & & & & & & & & & & & & 1 & 0 & 0,129 & $-0,037$ \\
\hline \multicolumn{5}{|c|}{ Harvard Step Test - Pulse Tíme - 3 Minutes of Restoration } & & & & & & & & & & & 0 & 1 & $-0,258$ & $-0,104$ \\
\hline Cooper Test & & & & & & & & & & & & & & & & & 1 & $585^{* 4}$ \\
\hline Beep test & & & & & & & & & & & & & & & & & & 1 \\
\hline
\end{tabular}

**. Correlation is significant at the 0.01 level (2-tailed).

*. Correlation is significant at the 0.05 level (2-tailed).

Table 4. Correlation analysis EG second statement

\begin{tabular}{|c|c|c|c|c|c|c|c|c|c|c|c|c|c|c|c|c|c|c|}
\hline Jump & engthe & rtialjum & Deadift & namome & ing to 00 & bbell holk & Plank & edical uni & medical & vittle $10 \times 1$ & hups to de & al presse. & se ing es: & Fest - Puls & est-Puls & e Time-3 & 3 Fooper Te: & Beep test \\
\hline Jump length of plecte & 1 & $6991^{\times x}$ & 0,264 & 0,111 & $479^{\times x}$ & $-0,061$ & 0,107 & 0,157 & 0,035 & $.0,165$ & $-0,07$ & 0,148 & $.0,005$ & $-0,182$ & $\cdot 0,167$ & $-0,23$ & $.442^{x x}$ & $340^{x}$ \\
\hline Vertialjump & & 1 & 0,327 & 0,071 & 0,261 & 0.245 & 0,268 & 0,236 & 0,293 & $-0,223$ & 0,146 & $409^{\circ}$ & 0,022 & $-0,285$ & $-0,143$ & $-0,163$ & 0,282 & 0,201 \\
\hline Deadift & & & 1 & $.469^{\times x}$ & 0,288 & 0,011 & $.471^{x x}$ & $.456^{x x}$ & $342^{x}$ & $-.453^{\times x}$ & 0,161 & $538^{\mathrm{xx}}$ & $.0,269$ & 0,007 & 0,06 & $-0,282$ & $380^{\circ}$ & $355^{x}$ \\
\hline Dynamometry & & & & 1 & $421^{x}$ & 0,113 & 0,094 & 0,219 & 0,214 & $.0,236$ & $378^{x}$ & 0,162 &,$- 374^{x}$ & 0,172 & 0,119 & $-345^{x}$ & $352^{x}$ & 0,153 \\
\hline Henging to o deniel & & & & & 1 & $-0,185$ & 0,259 & 0,238 & 0,105 & $-0,194$ & 0,056 & 0,155 & $-343^{x}$ & 0,3 & 0,088 & $-345^{x}$ & 0,199 & 0,122 \\
\hline Dumbbell hold $2 \mathrm{~kg}$ & & & & & & 1 & 0,275 & 0,221 & 0,216 & 0,023 & $.330^{x}$ & 0,188 & 0,125 & 0,06 & $-0,074$ & 0,069 & $356^{*}$ & 0,192 \\
\hline Plenk & & & & & & & 1 & 0,114 & 0,223 & $-0,295$ & 0,068 & $.621^{\mathrm{xx}}$ & $-0,183$ & 0,084 & 0,031 & $-0,205$ & 0,069 & 0,245 \\
\hline \multicolumn{3}{|c|}{ Throwing the me dical unit $(3 \mathrm{~kg})$ forward } & & & & & & 1 & $.434^{\mathrm{\alpha x}}$ &,$- 340^{\circ}$ & 0,035 & 0,051 & 0,113 & $\cdot 0,195$ & $-0,147$ & $-0,044$ & $.489^{\times x}$ & $390^{\times}$ \\
\hline \multicolumn{3}{|c|}{ Tossing of the medial unit (3kg) beck } & & & & & & & 1 & $-0,028$ & 0,066 & 0,199 & $-0,003$ & $-0,179$ & $-0,029$ & $-0,043$ & 0,288 & 0,221 \\
\hline Shuttle $10 \times 10 \mathrm{~m}$ & & & & & & & & & & 1 & $-0,101$ & $-0,294$ & 0,035 & 0,098 & 0,024 & 0,079 & $-0,265$ & $-0,218$ \\
\hline Pushups to denial & & & & & & & & & & & 1 & 0,153 & $.0,097$ & 0,111 & 0,14 & 0,056 & 0,253 & 0,118 \\
\hline \multicolumn{2}{|c|}{ Abdominal presses to denial } & & & & & & & & & & & 1 & $-0,187$ & $-0,099$ & $-0,106$ & $-394^{*}$ & 0,228 & $.452^{2 \times}$ \\
\hline \multicolumn{5}{|c|}{ Herverd Stap Test - pulse increase during the step test } & & & & & & & & & 1 & $-4588^{\circ \times}$ &,$- 723^{\times \times}$ & $363^{\circ}$ & $-0,037$ & $-0,047$ \\
\hline \multicolumn{4}{|c|}{ Herverd Steppe Test - Pulse after 1 minute } & & & & & & & & & & & 1 &, $650^{\times x}$ & 0,251 & $-0,134$ & $-0,213$ \\
\hline \multicolumn{4}{|c|}{ Herverd 5 teppe Test - Pulse ofter 3 minutes } & & & & & & & & & & & & 1 & $361^{x}$ & $-0,192$ & $-0,095$ \\
\hline \multicolumn{5}{|c|}{ Herverd Step Test - Pulse Time - 3 Minutes of Restoration } & & & & & & & & & & & & 1 & $-0,307$ & $-0,19$ \\
\hline Cooper Test & & & & & & & & & & & & & & & & & 1 & $704^{2 x}$ \\
\hline Eep test & & & & & & & & & & & & & & & & & & 1 \\
\hline
\end{tabular}

**. Correlation is significant at the 0.01 level (2-tailed).

*. Correlation is significant at the 0.05 level (2-tailed). 
After the required correlation analysis of the results of incoming and outgoing tests of the experimental group and the control group in order to visualize the significant correlation values of the movement qualities as shown in Figres 1, 2, 3 and 4.

They were picked up by the link with the image are accepted ( $\mathrm{P}^{\geq}$95\%). We numbered the tests of mobility as followes.
1 - jump length of place, 2 - vertical jump, 3 Stanova strength, 4 - dynamometer, 5 - Hanging to a denial, 6 - dumbbell hold $2 \mathrm{~kg}, 7$ - plank, 8 throwing the medical unit $(3 \mathrm{~kg})$ forward, 9 . Tossing of the medical unit $(3 \mathrm{~kg})$ back, $10-$ 10x10m shuttle, 11 - pushups to denial, 12 abdominal presses to denial, 13 - 3 minutes of Restoration, 14 - pulse increase during the step test, 15 - pulse retrieval 1 minute, 16 - pulse recovery 3 minutes, 17 - Cooper test, 18 - beep test.

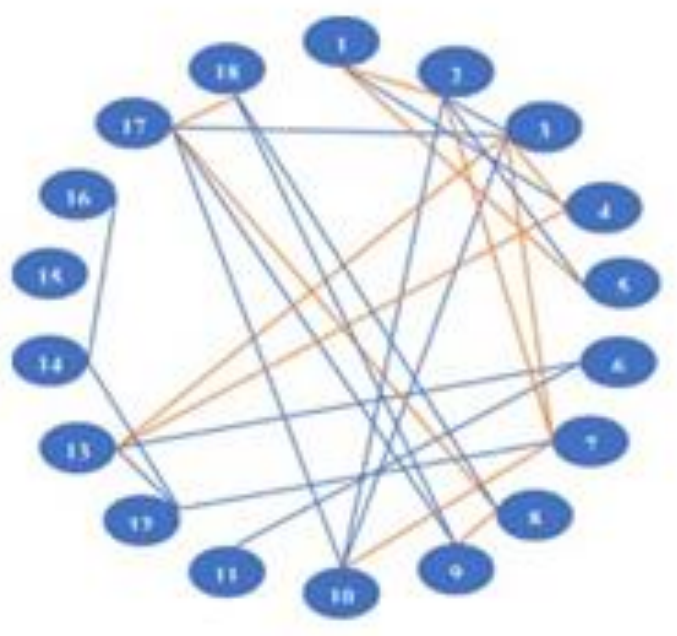

Fig. 1. Correlation structure of the relationship between the mobility rates of the EG on the incoming tests.

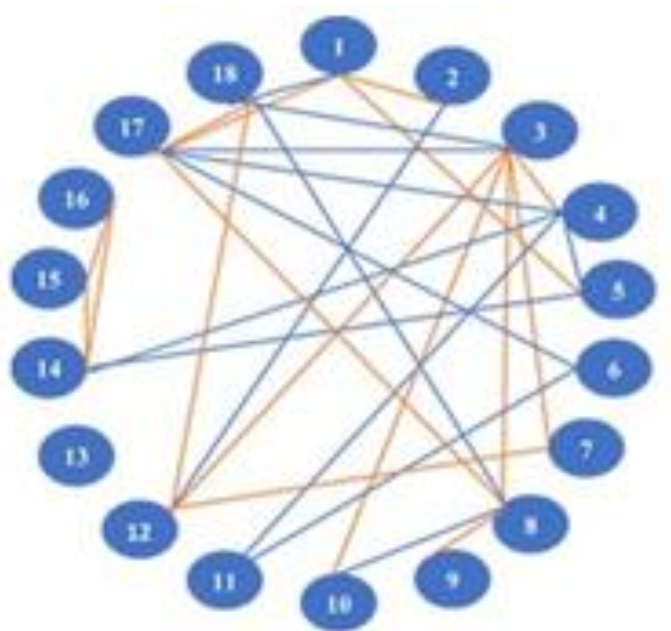

Note:

$95 \%$ Statistic persistence -

$99 \%$ Statistic persistence -

Fig. 2. Correlation structure of the relationship between the mobility rates of the EG of the outgoing tests. 
Note:

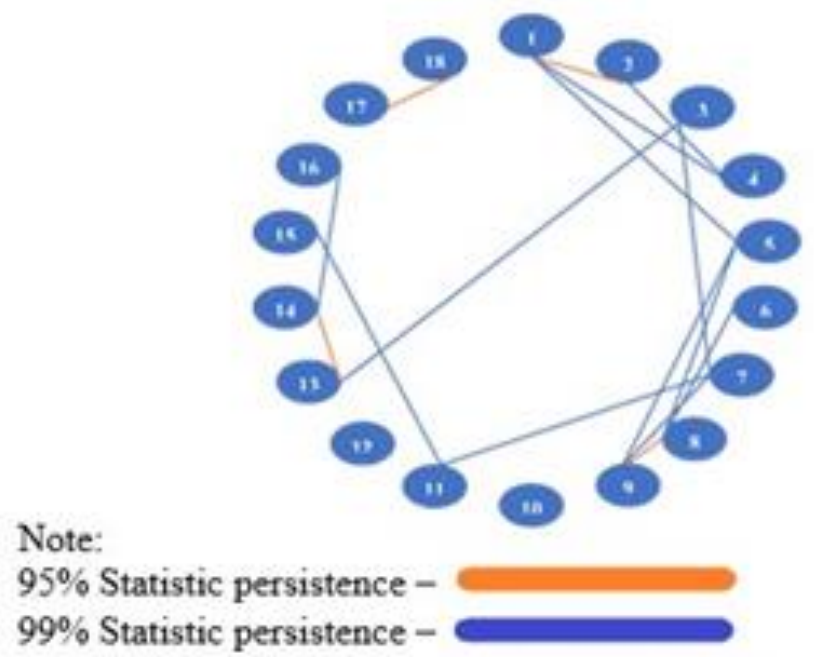

Fig. 3. Correlation structure of the relationship between the mobility rates of the $\mathrm{KG}$ on the incoming tests.

Note:

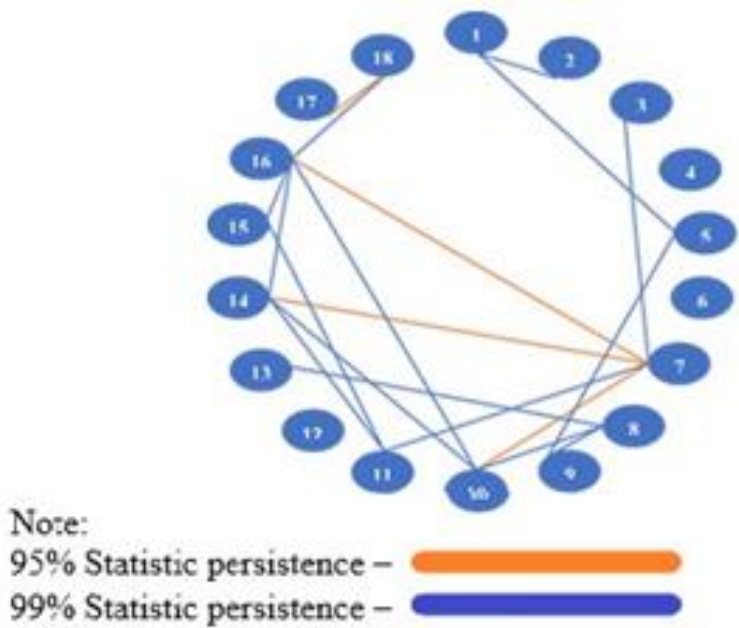

Fig. 4. Correlation structure of the relationship between the mobility rates of the KG of the outgoing tests.

After the need for an adequate civilian analysis, we can draw the following conclusions:

1. After applying the experimental fitness program, the links between muscle strength and endurance retain their key feature - with a significant number of reliable links. 2. Upon completion of the experiment, the statistically significant attributes of the EG increased (in the ingoing tests, they were 25, and in the rising ones, 30). Significantly increased links with $99 \%$ statistical confidence between traffic levels after using the author's fitness model.

3. In start tests, KG has 16 statistically tangible connections, and in the 19th century. 4. The study conducted showed the different degrees of correlation dependencies of the muscular strength and endurance of the students.

\section{REFERENCES}

1. Zhelyazkov, T., Endurance in Sport. Sofia, 2009.

2. Peltekova, I., "Comparative Analysis of the Program of Physical Education and Sport at the University of Bolgarhia and Kitaya". Sat. Kompetencje wspyiczesnego nauczyciela (Competence of the Modern Teacher) in order. by Tamara Zacharuk, Aneta Niewкgiowska, ISBN 978-83934036-9-1, Ed. Siedlce. Vol. 2, Poland, p. 146-151, 2012.

3. Peltekova, I., "Investigation of the Correlation Relationships Between Specific Condensing Qualities and the Accuracy of Shooting in a Basket". Scientific and Methodological Magazine "Vocational Education", book 4, year XIV, 2012, Ed. "I.Buki" - Ministry of Education, Youth and Sports. 
4. Peeva, D., Modern Trends in Education in Physical Education and Sports - European Perspective, Personality, Motivation, Sport; 978-954-718-521-0, 2018.

5. Peeva, D., Application of the Training Testing in the Theory and Methodology of Physical Education. Scientific Works Angel Kanchev University of Rousse. ISSN 1311-332, 2017.

6. Peeva, D., Kolev, N., "Investigation of the dynamics of the development of the basic physical qualities of the students of VTU" Todor Kableshkov "in Rugby Activities, Sport and Science Magazine, 1/2005, Sofia

7. Peeva, D., Kolev, N., "Dynamics of Development of Physical Quality of Strength of Students from VTU" Todor Kableshkov "After Systemic Rugby Activities", NSA, IV MNC, Sofia, 2007.

8. Dimitrova N., Information Technologies and Traffic Management in Sport, NSA PRESS, Sofia, 2007. Monograph.
9. Dimitrova N., "System Structure Analysis of Explosive Power", 2017. ; NSA PRESS; Sofia, ISBN 978-954-718-492-3 p.190-196, 2017.

10.Angelova, P., Therapeutic Stretching in Myofascial Pain, 2011, VTU "St. St. Cyril and Methodius", p. 260-265

11.Dyakova, G., Popova, M., Bodiprogram DG-MP - Methodology and Efficiency. "Angel Kanchev" RU, Scientific Works, T. 50, p. 8.2, Physical Education and Sports, ISSN 1311-3321, pp. 46-50, 2011.

12.Dyakova, G., Peeva, P., Nikolova, E., Bodifit training program - methodology and efficiency. Sp. Sports and Science, S., no. 3, pp. 29-36, 1997.

13.Gigova, V., Damianova, R., Statistical Methods in Sport - Guide for Students from the Bachelor's Degree of NSA, Sofia, 2012. 